Rev. Elev. Méd. vét. Pays trop., 1978, 31 (4) : 401-409.

\title{
Diagnose différentielle de l'espèce sur les carcasses et les abats des moutons et des chèvres de l'Afrique tropicale de l'Ouest
}

\author{
par P. CUQ $(*)$, J. ROZIER $(* *)$ et K. ADOMEFA $(* * *)$
}

\begin{abstract}
RESUMẼ
Les auteurs étudient, région par région, les caractères immédiats et médiats de la diagnose d'espèce des carcasses et des abats des petits ruminants de l'Afrique de l'Ovest. Ils montrent que la diagnose est facile lorsque la tête et la queue restent sur la carcasse ou la demi-carcasse et qu'elle est difficile lors. qu'elles en sont séparées. Ils distinguent trois types de caractères différentiels : ceux qui sont communs aux deux groupes régionaux, ceux qui sont particuliers aux espèces des régions tempérées et ceux qui sont propres aux ovins et aux caprins de l'Afrique de l'Ouest.
\end{abstract}

Les caractères anatomiques qui permettent la diagnose différentielle d'espèce des carcasses des moutons et des chèvres des régions tempérées sont bien connus depuis la publication du travail fondamental de LESBRE et PANISSET (1901). Il en va différemment pour les ovins et les caprins d'Afrique tropicale qui sont morphologiquement très proches. L'étude systématique des caractères spécifiques qui les concernent montre qu'une partie seulement des critères retenus pour les espèces européennes leur sont applicables et qu'ils présentent aussi des caractères différentiels qui leur sont propres.

\section{MATERIEL ET MÉTHODE}

Le travail a été réalisé à partir de 10 moutons et 10 chèvres qui ont été disséqués au Laboratoire d'Anatomie de l'E. I. S. M. V. et de plus

(*) Professeur, Chef du Service d'Anatomie, Histologie et Embryologie. Ecole Inter-Etats des Sciences et Médecine Vétérinaires de Dakar, Sénégal.

(**) Professeur, Chef du Service d'H. I. A. O. A. Ecole Nationale Vétérinaire d'Alfort, France.

$(* * *)$ Docteur Vétérinaire. Service National de l'Elevage. Lomé, Togo. de six cents carcasses ou demi-carcasses de petits ruminants qui ont été examinées à l'abattoir de Dakar (*). La collection d'Ostéologie du Laboratoire d'Anatomie de l'E. I. S. M. V. a aussi été utilisée.

En Afrique de l'Ouest seules les races ovines et caprines de grand format et à rendement important font l'objet d' " abattages contrôlés ». Les autres, plus petites et d'un rendement plus faible, sont habituellement consommées ou commercialisées sur place (abattages familiaux et abattages clandestins); la diagnose d'espèce ne se pose pas pour elles; le plus souvent, le consommateur ou l'acheteur connaît l'animal et participe à la saignée et au découpage. Nous avons donc limité cette étude aux seules races de grande taille qui font l'objet d'abattages contrôlés, soit, pour l'abattoir de Dakar, les moutons de races Maure, Peulh et Touareg et les caprins du Sahel.

En Afrique, les techniques de préparation des carcasses sont très variables d'un pays à

$\left(^{*}\right)$ Nous remercions son Directeur, Monsieur DIAWARA qui a bien voulu nous faciliter l'accès à l'entrepôt des carcasses. 
l'autre, voire d'une région à l'autre; la tête et la queue peuvent ainsi être sectionnées ou rester sur la carcasse ou la demi-carcasse ; l'ensemble trachée-poumon-cœur, œsophage, diaphragme et foie, peut aussi rester en place ou être prélevé en bloc; au Togo, la chèvre est souvent cuite avec sa peau. Il en est de même pour la découpe de détail qui varie pratiquement avec chaque opérateur. C'est pourquoi nous avons renoncé à rendre compte des résultats que nous avons obtenus en suivant un plan inspiré des stades successifs de la préparation de la viande et décidé de baser notre exposé sur les régions du corps.

Pour chacune de ces régions, nous indiquerons d'abord les caractères spécifiques immédiatement observables sur les deux faces, externe et interne, de la carcasse. Nous les compléterons ensuite par une série de caractères médiats qui nécessitent des manipulations de mise en évìdence et ne sont donc pas utilisables dans les conditions habituelles des circuits commerciaux.

De plus, pour faciliter la lecture du travail, des caractères typographiques différents sont utilisés selon la nature des caractères spécifiques rapportés : « Romain » ordinaire pour les critères de diagnose valables à la fois pour les petits ruminants d'Europe et pour ceux de l'Afrique de l'Ouest, «Italique » pour les critères valables en Europe seulement et « caractères gras romains " pour ceux qui sont particuliers aux petits ruminants de l'Afrique de l'Ouest.

\section{I. - PARTICULARITÉS SPÉCIFIQUES DE LA TÊTE DES PETITS RUMINANTS DE L'AFRIQUE DE L'OUEST}

Selon la méthode de préparation, la tête entière (ou fendue en deux) reste sur la carcasse (ou la demi-carcasse) ou est sectionnée au niveau de l'articulation atlanto-occipitale aussitôt après le dépouillement.

Selon le plan prévu, nous examinerons successivement les caractères spécifiques sur la face externe des têtes (têtes entières fixées aux carcasses), sur leur face interne (section médiane des têtes et têtes isolées) et les caractères médiats qui nécessitent une manipulation (caractères complémentaires).

\section{I.A. - DIAGNOSE DE L'ESPẼCE SUR LA TETE ENTIÉRE}

Dans son ensemble, la tête des petits ruminants est l'une des régions les plus propices à la diagnose de l'espèce. Les caractères que nous avons retenus sont valables en Europe comme en Afrique.

a) LE PROFIL DE LA TÊTE des ovins est toujours très convexe et leur chanfrein est bombé. La convexité du profil de la tête des caprins est beaucoup moins marquée et leur chanfrein est rectiligne.

b) LA SURFACE PARIÉTALE, bien visible après dépouillement, est large chez les ovins (6 à $7 \mathrm{~cm}$ d'après des mesures prises sur des moutons adultes à l'abattoir de Dakar) et les fosses temporales sont étroites et rejetées sur les faces latérales de la tête. Chez les caprins, au contraire, les fosses temporales empiètent sur la face dorsale de la tête : la surface pariétale est plus étroite ( 3 à $4 \mathrm{~cm}$ seulement d'après les mesures que nous avons effectuées dans les mêmes conditions que précédemment).

c) LES CORNES et leurs CHEVILLES OSSEUSES sont implantées plus près du plan médian et plus loin de l'orbite chez les ovins que chez les caprins. Dans ces derniers, les cornes sont franchement orientées vers l'arrière alors qu'elles divergent latéralement chez les ovins.

d) LE LARMIER est un cul-de-sac cutané qui repose dans la fosse larmière de l'os lacrymal des seuls moutons. Il est enlevé avec la peau, lors de la préparation des carcasses, mais il en subsiste toujours quelques fragments.

e) L'ANGLE DE LA MANDIBULE de la chèvre est à peu près droit $\left(90^{\circ}\right)$, il est obtu chez le mouton.

f) LA PORTION STERNO-ZYGOMATIQUE DU MUSCLE STERNO-CÉPHALIQUE est constante chez les caprins de toutes provenances où elle recouvre les bords inférieurs du muscle masseter et du corps de la mandibule. Elle est inconstante et souvent réduite à un grêle tractus fibreux dans les ovins des régions tempérées. Elle est toujours absente dans les moutons de l'Afrique de l'Ouest que nous avons examinés.

\section{I.B. - DIAGNOSE DE L'ESPËCE APRẼS SECTION MÉDIANE DE LA TETE}

La tête est souvent sectionnée médianement en même temps que la carcasse. Les PAPILLES JUGALES sont alors bien visibles : pour les espèces africaines seulement, elles sont plus 
grosses et moins nombreuses chez le mouton que chez la chèvre qui présente un grand nombre de fines papilles.

\section{I.C. - DIAGNOSE DE L'ESPẼCE SUR LA TETE ISOLEE}

Après section de l'articulation atlanto-occipitale, le TROU OCCIPITAL est visible. Pour les deux groupes régionaux, son bord supérieur (opisthion) est rectiligne chez le mouton et concave chez la chèvre tandis que son bord inférieur (basion) épais et arrondi chez les ovins est mince et tranchant chez la chèvre.

\section{I.D. - CARACTËRES COMPLEMENTAI- RES DE DIAGNOSE DE L'ESPËCE}

L'incision ou l'ablation de certains organes de la tête permettent de mettre en évidence d'autres caractères différentiels, tous valables à la foís pour les races africaines et pour les races européennes.

a) LE CONDUIT PAROTIDIEN peut être mis en évidence par section du muscle peaucier de la tête. Chez le mouton, il croise superficiellement et à mi-hauteur le muscle masseter. Chez la chèvre, il est toujours situé plus ventralement, près du bord inférieur de la mandibule.

b) LE CONDUIT AUDITIF EXTERNE du mouton est plus étroit que celui de la chèvre. D'après les mesures que nous avons prises, son diamètre est toujours inférieur à $0,6 \mathrm{~cm}$ chez celle-ci et toujours supérieur à $0,9 \mathrm{~cm}$ chez celui-là.

c) LA BULLE TYMPANIQUE de la chèvre est plus volumineuse que celle du mouton. Ventralement, elle dépasse le niveau de la portion basilaire de l'occipital chez les caprins alors qu'elle est toujours située au-dessus de ce plan chez les ovins.

d) LES CHOANES de la chèvre sont plus larges que ceux du mouton.

e) LA SUTURE LAMBDOÏDE ou SUTURE PARIÉTO-OCCIPITALE est transversale et rectiligne chez les ovins; elle forme un angle saillant vers l'avant chez les caprins.

f) Inversement, la SUTURE CORONALE ou SUTURE PARIÉTO-FRONTALE est rectiligne et transversale chez les caprins et elle forme un angle saillant vers l'avant dans les ovins. g) La SUTURE FRONTO-NASALE des ovins est rectiligne et transversale ; elle forme un angle saillant vers l'arrière chez les caprins.

h) Les OS NASEAUX sont longs, larges convexes et unis rostralement en une pointe nasale simple chez les ovins. Chez les caprins, ils sont courts, étroits, rectilignes et restent séparés oralement pour former une pointe nasale bifide.

i) L'APOPHYSE NASALE DE L'OS INCISIF est longue et atteint l'os nasal chez la chèvre ; elle est courte et ne l'atteint jamais chez le mouton. Pour cette raison et en relation avec la longueur des os naseaux, l'ÉCHANCRURE NASO-INCISIVE du mouton est plus profonde que celle de la chèvre.

j) A l'union naso-lacrymale, il existe une FONTANELLE constante chez les caprins. Elle est très rare chez les ovins.

k) La FENTE INCISIVE du mouton est plus longue (d'après nos mesures 2,5 à $3 \mathrm{~cm}$ ) que celle de la chèvre $(1,5$ à $2,2 \mathrm{~cm})$.

Par le nombre important des caractères spécifiques dont elle est le siège, la tête permet donc une diagnose facile de l'origine de la carcasse mais il faut qu'elle y reste adhérente.

\section{I1. - PARTICULARITÉS SPÉCIFIQUES DE LA QUEUE DES PETITS RUMINANTS DE L'AFRIQUE DE L'OUEST}

La QUEUE de la chèvre est courte (12 à $17 \mathrm{~cm}$ selon les mesures que nous avons effectuées sur des carcasses d'animaux adultes à l'abattoir de Dakar) et sa racine est aplatie dorso-ventralement. Celle du mouton est longue (30 à $39 \mathrm{~cm}$ ) et cylindrique.

La formule vertébrale confirme le caractère de longueur : la queue du mouton est formée de 17 à 22 vertèbres coccygiennes, celle de la chèvre de 11 à 13 seulement.

Au même titre que la tête, la queue est donc un très bon élément de la diagnose d'espèce des carcasses des petits ruminants. Mais l'une et l'autre sont malheureusement souvent séparées des carcasses ou des demi-carcasses et la diagnose doit alors tenir compte des caractères spécifiques de l'encolure, du troncet des membres sectionnés au niveau de l'interligne basipodemétapode. 

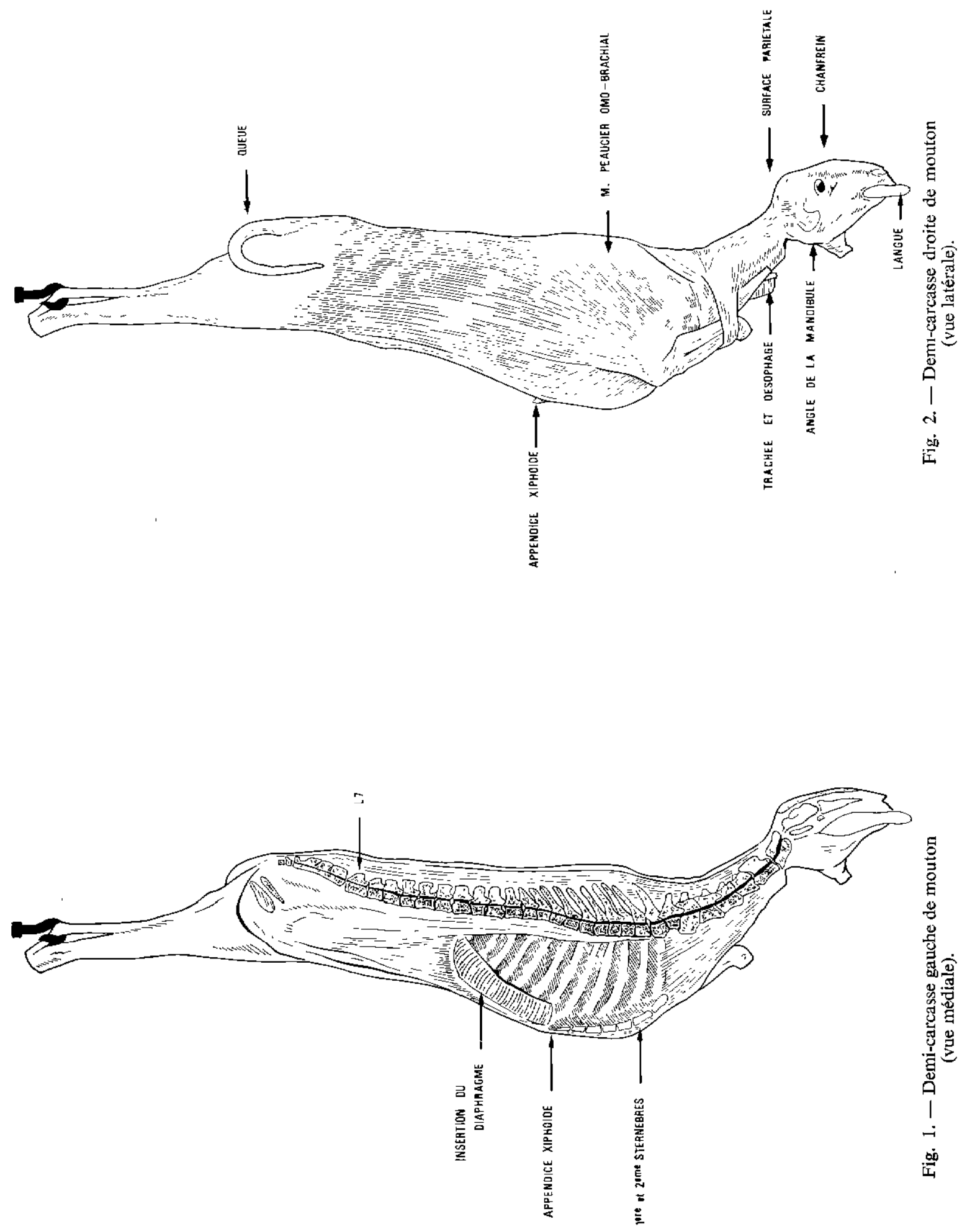


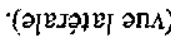

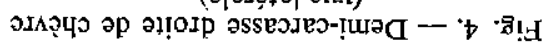

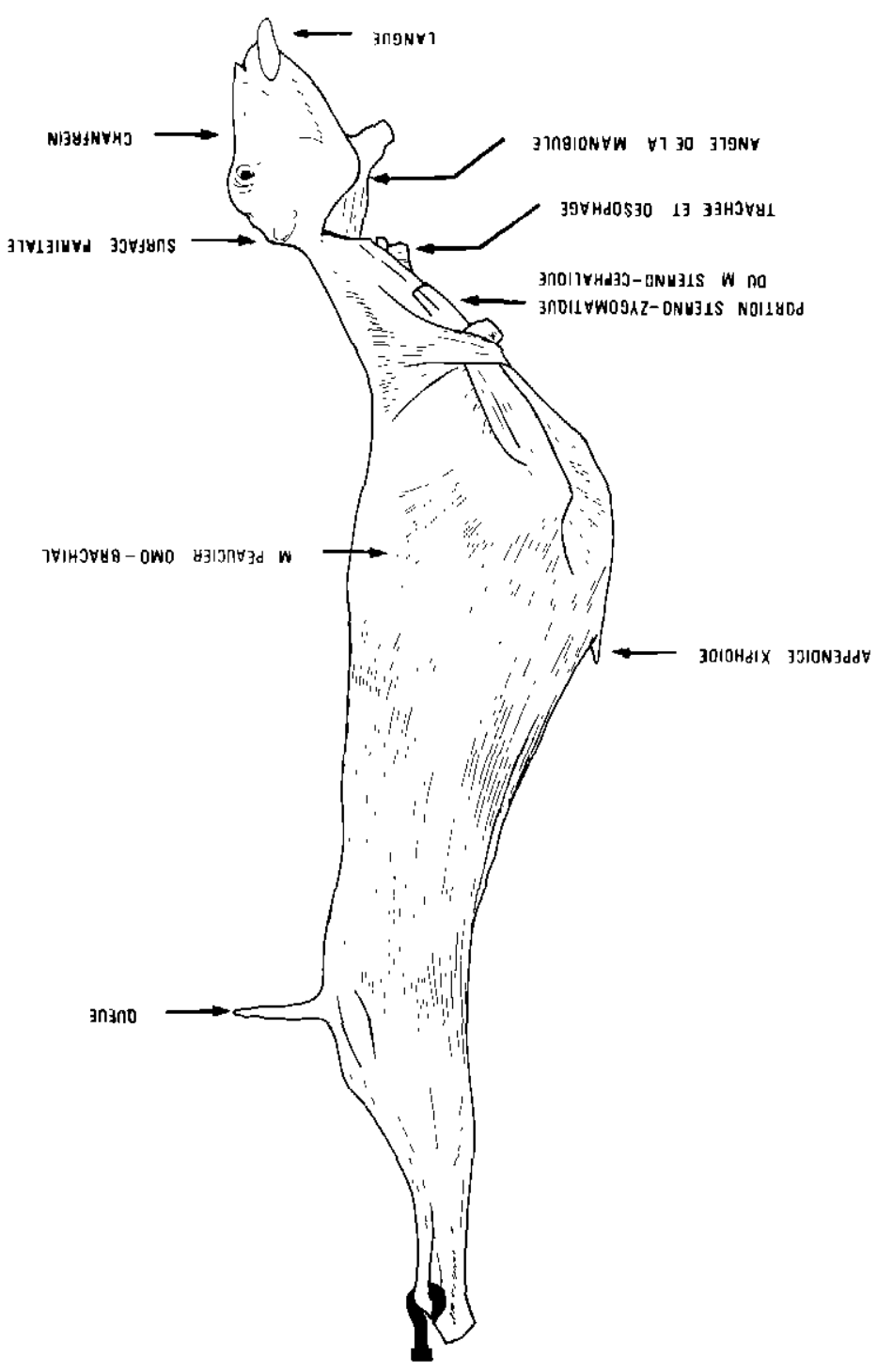

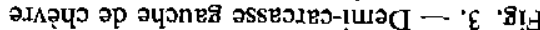

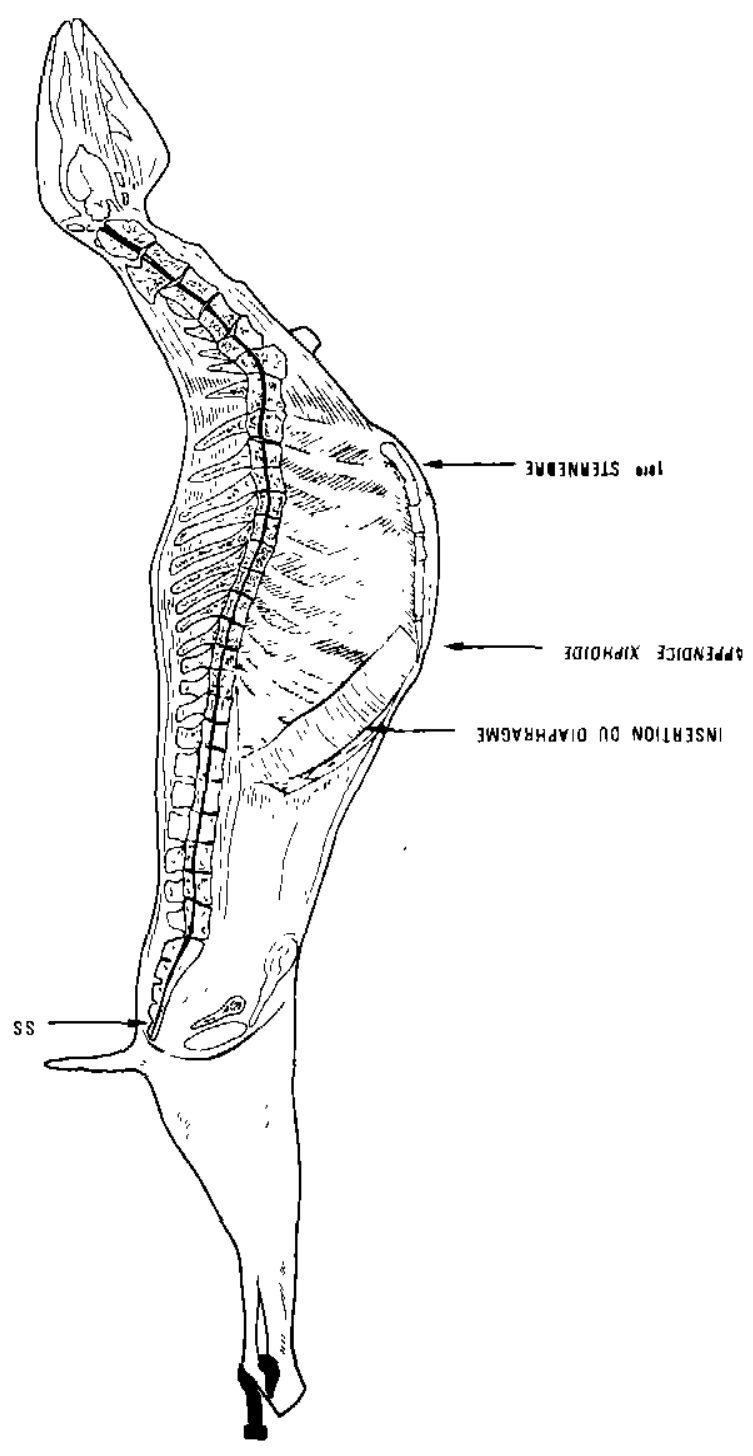




\section{III. - PARTICULARITÉS SPÉCIFIQUES DE L'ENCOLURE DES PETITS RUMI- NANTS DE L'AFRIQUE DE L'OUEST}

a) LES PROPORTIONS DE L'ENCOLURE, plus longue et plus grêle chez la chèvre, sont un bon élément de diagnose pour les races d'Europe. En Afrique Ies deux espèces ont une encolure de type caprin.

b) L'absence constante de Ia portion sternozygomatique du muscle sterno-céphalique des moutons africains que nous avons déjà signalée dans la région de la tête est aussi un bon caractère de diagnose pour l'encolure.

c) Lorsque la tête est séparée de l'encolure, l'ATLAS devient visible. Dans les deux groupes ethriques, le bord latéral de son alle est plus convexe chez le mouton; l'échancrure crâniale de son arc dorsal est large et taillée en $\mathrm{V}$ chez la chèvre, plus étroite et à fond rectiligne chez le mouton et son tubercule dorsal court et à sommet arrondi chez la chèvre est plus haut et plus pointu chez le mouton.

d) Sur la face interne de la demi-carcasse, la section des vertèbres cervicales est visible. Le sommet de l'APOPHYSE ÉPINEUSE DE L'AXIS présente un prolongement rostral qui dépasse crânialement le niveau du corps de la vertèbre chez la chèvre mais reste en deçà chez le mouton. Les APOPHYSES ÉPINEUSES DES VERTĖBRES CERVICALES SUIVANTES sont comprimées latéro-latéralement et perpendiculaires à l'axe de la vertèbre chez les ovins; elles sont en antéro-version chez les caprins.

Sur l'encolure des petits ruminants de l'Afrique de l'Ouest, l'espèce ne peut être déterminée qu'à partir des vertèbres et du muscle sterno-céphalique.

\section{IV. - PARTICULARITÉS SPÉCIFIQUES DU TRONC DES PETITS RUMINANTS DE L'AFRIQUE DE L'OUEST}

\section{IV.A. - PARTICULARITÉS SPECIFIQUES DU TRONC SUR LA FACE EX- TERNE DE LA CARCASSE}

1) La conformation générale du tronc (breviligne chez les ovins, longiligne chez les caprins), les proportions de la cage thoracique (plus profonde et plus plate chez la chèvre), le garrot (plus saillant chez les caprins) et la forme de la croupe (plus courte et plus large chez le mouton) sont de bons caractères de diagnose d'espèce des carcasses des petits ruminants des régions tempérées. En Afrique de l'Ouest les deux espèces sont du type caprin.

De même, la coloration générale des muscles (plus foncés chez le mouton), celle de la graisse de couverture (abondante chez les ovins et pratiquement absente chez les caprins), valables en Europe ne peuvent suffir à déterminer l'espèce pour les races africaines : les muscles sont souvent de couleur très voisine et leur teinte varie beaucoup avec le temps qui s'est écoulé depuis l'abattage, de même la graisse de couverture est rare, se dépose toujours dans les mêmes régions (rein et base de la queue) et a une couleur voisine dans les deux espèces.

2) La conformation de l'APPENDICE XIPHOIDE (large chez les caprins, petit, étroit et rond chez les ovins) est par contre un bon caractère de diagnose en Europe comme en Afrique.

3) Une particularité de la face externe de la carcasse est propre aux petits ruminants de l'Afrique de l'Ouest : le MUSCLE PEAUCIER OMO-BRACHIAL qui recouvre l'épaule a une limite dorsale rectiligne chez les caprins, convexe chez les ovins.

\section{IV.B. - PARTICULARITESS SPÉCIFIQUES DU TRONC SUR LA FACE INTER- NE DE LA DEMI-CARCASSE}

1) La forme des côtes (plus larges et plus courtes chez le mouton) n'est utilisable qu'en Europe. En Afrique les denx espèces sont du type caprin.

2) Tous les autres caractères de diagnose de la face interne de la demi-carcasse sont par contre valables dans les deux groupes ethniques.

a) Le DIAPHRAGME s'insère sur la dernière côte chez les ovins, un peu plus en avant chez les caprins.

b) La FORMULE VERTÉBRALE n'est pas la même dans les deux espèces : $7 \mathrm{~L}$ correspond toujours à un mouton, $5 \mathrm{~S}$ à une chèvre et l'association $6 \mathrm{~L}$ et $4 \mathrm{~S}$ est commune aux deux. 
c) Le STERNUM de la chèvre, étroit, allongé et rectiligne est formé de 7 sternèbres dont la première est seule oblique dorso-crânialement ; celui du mouton, plat, court et relevé comprend 6 ou 7 sternèbres dont la première est épaisse et les deux premières obliques dorso-crânialement.

\section{IV.C. - CARACTËRES COMPLEMENTAL- RES DE DIAGNOSE DE L'ESPECCE}

1) Certains caractères myologiques (section circulaire du muscle long dorsal des caprins, brièveté du muscle long costal des ovins, forte insertion costale du muscle petit psoas des caprins et étroitesse de la portion charnue du muscle grand oblique de l'abdomen) ne sont valables que pour les races européennes.

2) Pour être observé, le MUSCLE SCALÈNE SUPRA-COSTAL nécessite le lever de l'épaule. Il est toujours présent chez la chèvre. Il est inconstant et souvent réduit dans le mouton européen mais il est toujours absent dans les moutons de l'Afrique de l'Ouest.

3) Les caractères différentiels ostéologiques sont valables pour toutes les races des deux espèces.

a) Les APOPHYSES TRANSVERSES DES VERTĖBRES LOMBAIRES, courtes et relevées chez les ovins sont obliques vers le bas et se terminent en crochet chez les caprins.

b) L'AILE DE L'ILIUM est plus large et son col plus court chez les ovins que chez les caprins.

c) La face ventrale du SACRUM des caprins est marquée d'une crête médiane et ses bords latéraux sont minces et rabattus ventralement. Le sacrum des ovins est dépourvu de crête ventrale et ses bords latéraux sont épais et non rabattus ventralement.

\section{V. - PARTICULARITÉS SPÉCIFIQUES DU MEMBRE THORACIQUE DES PETITS RUMINANTS DE L'AFRIQUE DE L'OUEST}

Pour le membre thoracique, certains caractères spécifiques peuvent être observés sur la carcasse intacte, d'autres nécessitent des mises à jour.

\section{V.A. - PARTICULARITESS SPÉCIFIQUES DU MEMBRE THORACIQUE EN PLACE}

1) Les proportions de l'épaule (plus courte chez les ovins) et du bras (plus long que l'avantbras chez les caprins) ne sont utilisables que pour les petits ruminants des régions tempérées.

2) Par contre, en Afrique, la face distale des os de la deuxième rangée du carpe (visibles après la section du « pied ») permet la diagnose d'espèce : le capitato-trapézoide de la chèvre est plus large que celui du mouton, il présente un prolongement dorsal qui n'existe pas dans ce dernier.

\section{V.B. - CARACTĖRES COMPLEMEN- TAIRES DE DIAGNOSE DE L'ES- PÉCE}

1) Les caractères complémentaires myologiques valables en Europe (adhérence du muscle féchisseur superficiel des doigts et de l'ulnaire médial et absence constante du muscle de Thiernesse chez la chèvre) ne peuvent pas être utilisés en Afrique: dans les deux espèces, le muscle de Thiernesse est présent et le muscle fléchisseur superficiel des doigts adhère à l'ulnaire médial.

2) Les caractères complémentaires ostéologiques sont par contre valables dans les deux zones climatiques;

a) La SCAPULA des ovins est plus courte et plus large que celle des caprins, son épine scapulaire est incurvée et tubéreuse alors qu'elle est rectiligne et tranchante chez la chèvre.

b) L'ESPACE INTER-OSSEUX PROXIMAL DES OS DE L'AVANT-BRAS est plus proche de l'extrémité supérieure du radius et plus étroit chez la chèvre. Au-dessus d'elle, le radius et l'ulna sont intimement soudés dans les caprins, alors que la soudure des deux os est incomplète chez les ovins.

\section{VI. - PARTICULARITÉS SPÉCIFIQUES DU MEMBRE PELVIEN DES PETITS RUMINANTS DE L'AFRIQUE DE L'OUEST}

\section{VI.A. - PARTICULARITES SPECIFIQUES DU MEMBRE PELVIEN EN PLACE}

Les proportions du bassin et de la cuisse (plus longiligne chez la chèvre) les rapports entre les muscles ischio-tibiaux et gastrocnémiens (dont 
es ventres sont entièrement cachés chez le mouton seulement) sont inutilisables pour les espèces africaines, toutes deux du type caprin.

\section{VI.B. - CARACTẼRES TAIRES}

COMPLEMEN-

Tous les caractères complémentaires sont osseux et à rechercher par incision des masses musculaires.

a) A l'exception du fémur de la chèvre qui, dans les espèces européennes, est plus long que celui du mouton (en Afrique, les deux espèces sont du type caprin) tous les autres caractères sont valables dans les deux régions climatiques.

b) Le COL DU FÉMUR est plus large et plus court et la FOSSE TROCHANTÉRIQUE plus étroite et plus profonde chez le mouton.

c) La TROCHLEE FÉMORALE de la chèvre est longue et étroite, sa lèvre latérale est plus haute que la médiale et sa gorge présente souvent une fossette synoviale. Celle du mouton est plus courte et plus large, ses lèvres sont égales et sa gorge est toujours dépourvue de fossette synoviale.

d) Le TIBIA du mouton est rectiligne, celui de la chèvre est légèrement contourné en $S$ à convexité proximale médiale.

e) La CRÈTE TIBIALE de la chèvre est plus courte que celle du mouton.

f) Le CALCANEUS de la chèvre est plus haut que celui du mouton. Il est aussi un peu incurvé médialement.

\section{VII. - PARTICULARITÉS SPÉCIFIQUES DES ABATS DES PETITS RUMINANTS DE L'AFRIQUE DE L'OUEST}

La diagnose d'espèce peut aussi être effectuée sur certains abats que nous étudierons par appareil.

\section{VUI.A. - ABATS DE L'APPAREIL DIGESTIF}

1) La LANGUE de la chèvre est plus longue et plus étroite que celle du mouton et les PAPILLES CALICIFORMES de ce dernier sont plus larges et plus étroites.

2) Le DIAMÈTRE DE L'OESOPHAGE est supérieur à celui de la trachée chez les ovins. Les deux sont égaux chez les caprins.
3) La VESSIE CONIQUE CAUDO-VENTRALE DU RUMEN de la chèvre est plus développée que la caudo-dorsale et les sillons coronaires qui circonscrivent leurs bases ne sont pas au même niveau. Les vessies coniques $\mathrm{du}$ rumen du mouton sont égales et les deux sillons coronaires sont disposés symétriquement.

4) Les PAPILLES DU RUMEN de la chèvre sont plus nombreuses et plus grosses que celles du mouton.

5) Le volume du RÉSEAU est supérieur à celui du FEUILLET chez les ovins; les deux organes sont égaux chez les caprins. En Europe les crêtes du réseau sont plus hautes et plus nombreuses chez la chèvre que chez le mouton. Elles sont de même hauteur dans les espèces africaines.

6) Les PLIS SPIRALES INEFFACABLES DE LA CAILLETTE sont plus nombreux chez la chèvre que chez le mouton. Pour les races africaines, nous en avons dénombré 20 chez les caprins et 15 à 17 chez les ovins.

7) En Europe, le bulbe duodénal est absent chez la chèvre et bien développé chez le mouton et l'intestin de ce dernier est de calibre plus réduit. En Afrique ces caractères sont inutilisables, le bulbe duodénal est peu développé dans les caprins et les ovins dont les intestins sont de calibres voisins.

8) Le foie de la chèvre européenne est plus étroit et plus long que celui du mouton. Ce caractère n'est pas net pour les espèces africaines dans lesquelles cependant, comme en Europe, le SILLON DU LIGAMENT ROND est plus long chez la chèvre et l'ANGLE FORMÉ PAR LE CONDUIT CYSTIQUE ET LE CONDUIT HÉPATIQUE COMMUN est droit dans les caprins et aigu dans les ovins.

\section{VII.B. - APPAREIL RESPIRATOIRE}

Dans les deux régions, le poumon droit du mouton présente un lobe moyen crânial qui n'est pas individualisé chez la chèvre. Dans cette dernière, en outre, la scissure inter-lobaire crâniale $G$ ou $D$, est moins profonde et le lobe moyen gauche plus développé.

\section{VII.C. - LE COEUR}

1) Le trabécule septo-marginal du mouton d'Europe a un diamètre inférieur à celui de la chèvre. En Afrique, sa dimension est la même dans les deux espèces. 
2) Par contre, dans les deux zones climatiques, le SILLON DU BORD CAUDAL DU CEEUR est rectiligne chez les caprins et dévié à gauche chez les ovins.

\section{VII.D. - LA RATE}

La RATE du mouton est plutôt triangulaire et celle de la chèvre plutôt quadrangulaire.

\section{CONCLUSION}

La diagnose d'espèce de la carcasse des petits ruminants de l'Afrique de l'Ouest est facile lorsque la tête ou la queue n'ont pas été sectionnées.

Dans le cas contraire, la diagnose reste possible mais est plus difficile que dans les espèces européennes qui présentent d'importants caractères différentiels qui leur sont propres : proportions de l'encolure, du tronc dans son ensemble, de la cage thoracique, de la croupe, de l'épaule, du bras, de l'avant-bras et de la cuisse, saillie du garrot, coloration des muscles et de la graisse de couverture, forme des côtes et des muscles long dorsal, petit psoas et grand oblique de l'abdomen, adhérence du muscle fléchisseur superficiel des doigts et du muscle ulnaire médial, présence ou absence du muscle de Thiernesse, rapports des muscles ischio-tibiaux et gastrocnémiens et longueur du fémur.

Certains caractères de diagnose sont, par contre, particuliers aux espèces africaines : nombre et dimension des papilles jugales, absence constante de la portion sterno-zygomatique du muscle sterno-céphalique et du muscle scalène supra-costal chez le mouton, contour dorsal du muscle peaucier omo-brachial et forme de la face distale de l'os capitato-trapézoïde.

Tous les autres caractères que nous avons étudiés sont valables dans les deux groupes ethniques

Pour les abats, à l'exception de la hauteur et du nombre des crêtes du réseau, de la forme du bulbe duodénal, du calibre de l'intestin et de la grosseur du trabécule septo-marginal du ventricule droit, tous les critères de diagnose utilisés en Europe sont applicables aux races africaines.

\section{SUMMARY}

Differential diagnosis on the carcasses and offals of sheep and goats in Tropical West Africa

The authors study, region by region, the immediate and mediate elements of the species diagnosis of carcasses and offals of small ruminants in West Africa. They show that the diagnosis is easy when the head and tail are left on the carcass or half-carcass, but it is difficult when they are apart. They note three types of differential characteristics : those, common to both regional groups, those particular to the species of temperate climates and those belonging to sheep and goats of West Africa.

\section{RESUMEN}

Diagnosis diferencial de la especie sobre los canales y los despojos de las ovejas $y$ eabras de Africa tropical

Los autores estudian región por regıón, los carácteres inmediatos y mediatos de la diagnosis de especies de las canales y de los despojos de los pequeños rumiantes de Afrıca del Oeste. Muestran que la diagnosis es fácil cuando la cabeza y la cola quedan sobre la canal o media canal y que es difícil cuando están separadas. Distinguen tres tıpos de carácteres diferenciales : los que son comunes a los dos grupos regionales, los que son particulares a las especies de las regiones templadas y los que son propios a los ovinos y a las cabras del Africa del oeste. 